\title{
On Orthonormal Wavelets and Paraunitary Filter Banks
}

\author{
Anand K. Soman, Student Member, IEEE, and P. P. Vaidyanathan, Fellow, IEEE
}

\begin{abstract}
Binary tree-structured filter banks have been employed in the past to generate wavelet bases. While the relation between paraunitary filter banks and orthonormal bases is known to some extent, there are some extensions which are either not known, or not published so far. In particular it is known that a binary tree-structured filter bank with the same paraunitary polyphase matrix on all levels generates an orthonormal basis. First, we generalize the result to binary trees having different paraunitary matrices on each level. Next, we prove a converse result: that every discrete-time orthonormal wavelet basis can be generated by a tree-structured filter bank having paraunitary polyphase matrices.

We then extend the concept of orthonormal bases to generalized (i.e., nonbinary) tree structures, and see that a close relationship exists between orthonormality and paraunitariness in this case too. We prove that a generalized tree structure with paraunitary polyphase matrices produces an orthonormal basis. Since not all bases can be generated by tree-structured filter banks, we prove that if an orthonormal basis can be generated using a tree structure, it can be generated specifically by a paraunitary tree.
\end{abstract}

\section{INTRODUCTION}

$\mathrm{R}^{\mathrm{H}}$ ECENTLY, wavelet transforms have evoked considerable interest in the signal processing community. They have found applications in several areas such as speech coding, edge detection, data compression, extraction of parameters for recognition and diagnostics, etc. [1]-[3]. Since wavelets provide a way to represent a signal on various degrees of resolution, they are a convenient tool for analysis and manipulation of data. In [4], Mallat describes a multiresolution algorithm for decomposing and reconstructing images. In [2], Mallat and Hwang have shown that the local maxima of the wavelet transform detect the location of irregular structures. They have also shown that it is possible to reconstruct one and two dimensional signals from the local maxima of their wavelet transform. Applications of wavelets to subband speech and image coding techniques can be found in [5]-[7]. Wavelets can also be used in the detection of transient signals [6]. Orthonormality is a very desirable property in several of these applications, and indeed, the problem of generating orthonormal wavelets is of considerable interest.

Manuscript received June 9, 1991; revised May 5, 1992. This work was supported in part by NSF Grant MIP 8919196, Tektronix Inc., and Rockwell International.

The authors are with the Department of Electrical Engineering, California Institute of Technology, Pasadena, CA 91125.

IEEE Log Number 9206026.
The theory of wavelets was originally developed in the context of continuous time functions [8], [9]. It has since been related to the familiar idea of quadrature mirror filter (QMF) banks. Continuous time wavelets can be obtained from infinite-level binary tree-structured QMF banks, with the same filters on each level [10]. This infinite recursion gives rise to two continuous time functions $\psi(t)$ and $\phi(t)$ which are termed as the wavelet function and the scaling function, respectively. The wavelet basis is then obtained by dyadic scaling and shifting of the wavelet function $\psi(t)$. It has been shown [10] that if this basis of continuous time functions is orthonormal, then the single QMF pair used to generate them is paraunitary [11].

Subsequently, the notion of wavelets has been extended to discrete time. This is more suitable in a number of signal processing applications. However, there appears to be no universal definition of wavelets in discrete time. Some authors have referred to a one-level paraunitary filter bank as wavelet transforms. This definition is, however, too restrictive. Probably the definition which best captures the notion of wavelets in discrete-time is the idea of having a binary tree with a finite number of levels, simultaneously allowing different filters on each level. This definition is fairly general, and is also useful from a practical viewpoint. We shall subscribe to this definition in this paper. The idea of wavelets in discrete time therefore reduces to that of a filter bank with dyadically increasing decimation ratios. This idea of a filter bank with dyadic decimation ratios can be generalized to filter banks with nonuniform, nondyadic decimation ratios. The basis functions corresponding to such nonuniform filter banks have been referred to as wavelet packets [1], [12]. One of the ways to realize such nonuniform filter banks is by using general tree structures.

Given the importance of orthonormal wavelets and wavelet packets in several applications, it becomes natural to seek necessary and sufficient conditions under which these discrete time basis functions are orthonormal. While the relation between orthonormal bases and paraunitary filter banks is known to some extent, there are some extensions which are either not known, or not published so far. There also appears to be no published work which can serve as a comprehensive reference for the generalized orthonormal wavelet bases (wavelet packets) and paraunitary filter banks. The aim here is to present a complete study of this relation. The following are the main points of this paper. 
1) Paraunitariness implies wavelet orthonormality. It is shown that if a binary tree is constructed using the same paraunitary block on each level, the resulting discrete time basis is orthonormal. A straightforward extension is that the discrete time basis continues to be orthonormal even if different paraunitary blocks are used on each level (Theorem 1).

2) Orthonormality implies paraunitariness. We prove that every orthonormal wavelet basis can be generated using binary tree structured filter banks with paraunitary building blocks (Theorem 2). The proof also shows how we can synthesize the tree, i.e., we can identify the filter pair on each level of the tree, starting from the given orthonormal basis. Furthermore, if an orthonormal wavelet basis is generated using a binary tree, the filters on each level have to be generalized paraunitary (i.e., paraunitary, except for constant scaling) (Corollary 1). These results allow us to generate all orthonormal wavelet bases simply by manipulating the coefficients of a set of lattice structures.

3) Orthonormality of wavelet packets. We develop the concept of orthonormality for nonuniform filter banks. In particular, we show that if $f_{k}(n)$ and $f_{i}(n)$ are two of the basis functions, then the orthonormality condition can be written as

$$
\sum_{n=-\infty}^{\infty} f_{k}(n) f_{l}^{*}\left(n-g_{l k} i\right)=\delta(k-l) \delta(i) .
$$

Here, $g_{l k}$ is the greatest common divisor (gcd) of $\left(I_{k}, I_{l}\right)$, the decimation factors corresponding to the two filters. The fact that the ged is involved in the definition has not been brought to attention before. We also prove that if a set of wavelet packets is realized using a general tree structure with paraunitary matrices on each level, the resulting basis is orthonormal (Theorem 3). Since not all bases can be generated using tree structures, the exact converse of this result is not true, unlike the binary case. However, if an orthonormal basis can be generated using a tree structure, we show that it can be generated specifically by a tree having paraunitary filters on each level (Theorem 4). This establishes the relation between paraunitariness and orthonormality in the case of wavelet packets.

\section{Nomenclature}

Boldfaced quantities denote matrices and vectors, as in $\boldsymbol{A}$ and $\boldsymbol{x} . \boldsymbol{A}^{T}$ denotes the transpose of the matrix $\boldsymbol{A}$. A superscript asterisk as in $f^{*}(n)$ denotes conjugation. The tilde notation as in $\tilde{H}(z)$ stands for conjugation of coefficients followed by transposition followed by replacing $z$ by $z^{-1}$. Consider a transfer function $A(z)$. It can be written in terms of its $M$ polyphase components [11] as follows:

$$
\begin{aligned}
A(z)= & a_{0}\left(z^{M}\right)+z^{-1} a_{1}\left(z^{M}\right) \\
& +\cdots+\left(z^{-M+1}\right) a_{M-1}\left(z^{M}\right) .
\end{aligned}
$$

This is known as type I polyphase. Let $H_{i}(z), i=0, \cdots$, $M-1$, be a set of analysis filters. They can be written as

$$
H_{k}(z)=\sum_{i=0}^{M-1} z^{-l} E_{k i}\left(z^{M}\right) \quad k=0, \cdots, M-1 .
$$

The matrix $\boldsymbol{E}(z)=\left[E_{k, l}\right]$ is called the polyphase matrix of the analysis filters. There is also a type II polyphase representation which is as follows:

$$
\begin{aligned}
A(z)= & z^{-M+1} a_{0}^{\prime}\left(z^{M}\right)+z^{-M+2} a_{1}^{\prime}\left(z^{M}\right) \\
& +\cdots+a_{M-1}^{\prime}\left(z^{M}\right) .
\end{aligned}
$$

Let $F_{i}(z), i=0, \cdots, M-1$, be a set of synthesis filters. They can be written as

$F_{k}(z)=\sum_{l=0}^{M-1} z^{-M+1+1} R_{l k}\left(z^{M}\right) \quad k=0, \cdots, M-1$.

The matrix $\boldsymbol{R}(z)=\left[R_{l, k}\right]$ is called the polyphase matrix of the synthesis filters.

If $a(n)$ is the inverse transform of $A(z)$, then $a(M n)$ is called the $M$-fold decimated version of $a(n)$. In the $z$-domain, we use a downward arrow to denote the decimation operator, for example, $(A(z)) \downarrow_{M}$ is the $z$ transform of the $M$-fold decimated version of $a(n)$. A matrix $E(z)$ is said to be paraunitary if it satisfies [11]

$$
\tilde{E}(z) E(z)=I \quad \text { for all } z .
$$

Given a set of $M$ filters $H_{k}(z), k=0, \cdots, M-1$, we can define an $M$ by $M$ polyphase matrix for these filters as in [11]. We say that the set of filters forms a paraunitary set (abbreviated PU-set) if their polyphase matrix is paraunitary. In particular, two filters with a paraunitary polyphase matrix are said to form a PU-pair.

The abbreviation "gcd" stands for the greatest common divisor. The abbreviation FIR stands for finite impulse response.

\section{Preliminaries}

In this section, we shall develop the background useful for dealing with the remainder of the paper. Most of what is presented in this section can be inferred from the work of Daubechies [10]. The filter-bank approach to wavelets has also been presented by Vetterli [12], [15] and by Vaidyanathan [16]. Our notation in this paper will be similar to that in [16].

The wavelet transform provides a time-scale representation of a signal which makes it possible to analyze signals on various degrees of resolution. It is a representation of a signal in terms of a peculiar set of orthonormal functions. The peculiarity of this orthonormal family is that it is obtained by shifting and dilating a single function, often termed as the mother wavelet. Let $x(t)$ be the signal under consideration. Mathematically, we can write its wavelet transform as

$$
X_{\mathrm{CWT}}(p, q)=\frac{1}{\sqrt{|p|}} \int_{-\infty}^{\infty} x(t) f\left(\frac{t-q}{p}\right) d t .
$$


$X_{\mathrm{CWT}}(p, q)$ is referred to as the continuous wavelet transform (CWT) of the signal $x(t)$. It is thus named because the variables $p$ and $q$ are continuous variables. Note that the family of functions $f((t-q) / p)$ is generated from a single function $f(t)$ by translations and dilations. $p$ is the dilation parameter, whereas $q$ is the translation parameter. This is a mapping from a one-dimensional continuous variable $t$, to a two dimensional continuous variable $(p, q)$. If we restrict $p$ and $q$ to take discrete values, we obtain a mapping from a one-dimensional continuous variable to a two-dimensional discrete variable. This is called the discrete wavelet transform (DWT).

In signal processing literature [15], [16], there has been defined a similar operation as the above for discrete time signals. We say,

$$
y_{k}(n)=\sum_{m} h_{k}(m) x\left(I_{k} n-m\right), \quad 0 \leq k \leq L
$$

is the discrete time wavelet transform (DTWT) of the signal $x(n)$. It is common to choose $I_{k}=2^{k+1}, k=0, \cdots$, $L-1$, and $I_{L}=2^{L}$. This is the binary DTWT, often referred to simply as the DTWT [15], [16]. The quantities $y_{k}(n), k=0, \cdots, L$ are called the wavelet coefficients of the signal $x(n)$. Equation (2.2) is a convolution followed by decimation by a factor $I_{k}$. The wavelet coefficients can hence be visualized as being obtained by passing the signal through a bank of $L+1$ filters $h_{k}(n)$, and decimating the filter outputs by factor $I_{k}$. The condition that the filter bank be a maximally decimated system implies that $\Sigma\left(1 / I_{k}\right)=1$. This viewpoint has been explained in [16]. Specifically, the binary DTWT (which we shall henceforth refer to as DTWT) can be obtained by passing the signal $x(n)$ through a binary tree-structured analysis bank (as shown in Fig. 1(a) for the case $L=3$ ). Consider the corresponding binary tree-structured synthesis bank shown in Fig. 1(b). It is well known [13] that it is possible to design such perfect-reconstruction tree-structured filter banks. For the perfect reconstruction system, the signal $x(n)$ can be recovered from its wavelet coefficients as

$$
x(n)=\sum_{k=0}^{L} \sum_{m} y_{k}(m) \eta_{k m}(n) .
$$

This is the "inverse" DTWT operation. The $\eta_{k m}(n)$ are termed the wavelet basis functions. The perfect reconstruction binary tree-structured analysis-synthesis system can be redrawn as a traditional filter bank as in Fig. 2(a). Fig. 2(b) shows the typical frequency responses of the analysis filters. Note that the amplitudes of the filters increases as the bandwidth decreases, keeping the energy in each of them equal. With reference to Fig. 2(a), it can be shown [15], [16] that the synthesis filters are related to the functions $\eta_{k m}(n)$ as

$$
\begin{aligned}
& \eta_{k m}(n)=f_{k}\left(n-2^{k+1} m\right), \quad k=0, \cdots, L-1 \\
& \eta_{L m}(n)=f_{L}\left(n-2^{L} m\right) .
\end{aligned}
$$

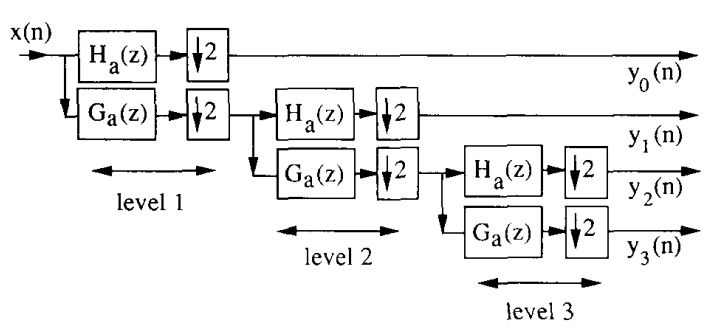

(a)

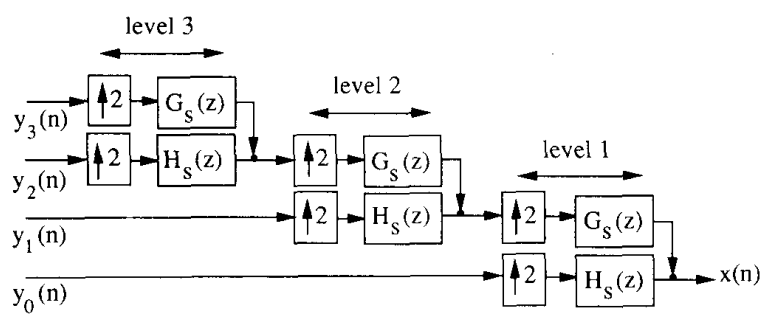

(b)

Fig. 1. A tree-structured filter bank used for generating wavelets. (a) The analysis bank. (b) The synthesis bank.

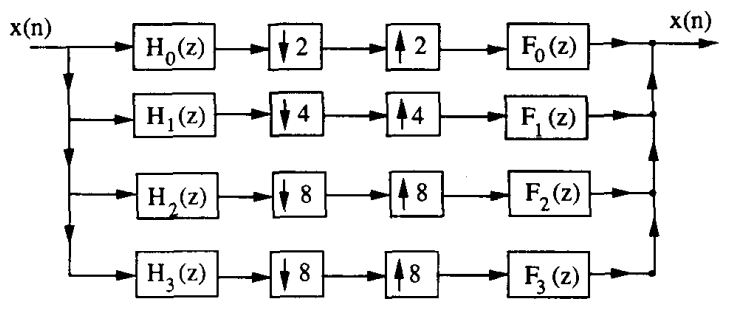

(a)

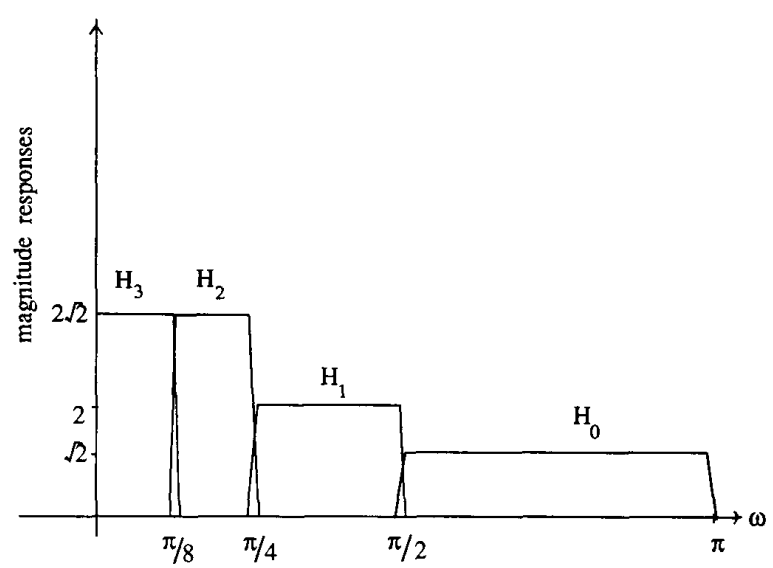

(b)

Fig. 2. (a) A four channel wavelet filter bank. (b) Typical appearances of magnitude responses of analysis filters in a three-level tree.

For perfect reconstruction systems, therefore, every signal can be represented in terms of a wavelet transform, and every signal can be recovered from its wavelet coefficients. Note that this is not the case with ordinary. Fourier transform. In fact, not all sequences have a Fourier transform! 


\section{Orthonormality}

The wavelet basis functions in (2.3) are said to be orthonormal if they satisfy the relation [10]

$$
\sum_{n} \eta_{k m}(n) \eta_{l i}^{*}(n)=\delta(k-l) \delta(m-i) .
$$

In terms of the filter responses in a binary tree-structured filter bank, this is equivalent to the condition

$$
\begin{aligned}
& \sum_{n} f_{k}\left(n-2^{k+1} m\right) f_{l}^{*}\left(n-2^{l+1} i\right)=\delta(k-l) \delta(m-i), \\
& \quad k, l=0, \cdots, L-1 \\
& \sum_{n} f_{L}\left(n-2^{L} m\right) f_{l}^{*}\left(n-2^{l+1} i\right)=0 \\
& \quad l=0, \cdots, L-1 \\
& \sum_{n} f_{L}\left(n-2^{L} m\right) f_{L}^{*}\left(n-2^{L} i\right)=\delta(m-i)
\end{aligned}
$$

With a change of variables this becomes

$$
\begin{aligned}
& \sum_{n} f_{k}(n) f_{l}^{*}\left(n-2^{l+1} i\right)=\delta(k-l) \delta(i) \\
& \quad l \leq k, \quad l, k=0, \cdots, L-1 \\
& \sum_{n} f_{L}(n) f_{l}^{*}\left(n-2^{l+1} i\right)=0, \quad l=0, \cdots, L-1 \\
& \sum_{n} f_{L}(n) f_{L}^{*}\left(n-2^{L} i\right)=\delta(i) .
\end{aligned}
$$

In the transform domain this is equivalent to

$$
\begin{aligned}
\left(F_{k}(z) \tilde{F}_{l}(z)\right) \downarrow_{2^{l} \cdot 1}= & \delta(k-l), \\
& l \leq k, \quad k, l=0, \cdots, L-1 \\
\left(F_{L}(z) \tilde{F}_{l}(z)\right) \downarrow_{2^{\prime-1}}=0, \quad l=0, \cdots, L-1 & (2.13) \\
\left(F_{L}(z) \tilde{F}_{L}(z)\right) \downarrow_{2^{L}}=1 . &
\end{aligned}
$$

First, consider the case of a one-level "tree" shown in Fig. 3(a), redrawn as in Fig. 3(b), where $R(z)$ is the polyphase matrix of the filters $F_{0}(z)$ and $F_{1}(z)$. It has been shown [16] that if the matrix $\boldsymbol{R}(z)$ is paraunitary, then the filters satisfy the orthonormality condition

$$
\begin{aligned}
& \sum_{n} f_{k}(n-2 m) f_{l}^{*}(n-2 i) \\
& \quad=\delta(k-l) \delta(m-i), \quad k, l=0,1 .
\end{aligned}
$$

In the $z$-domain, this becomes

$$
\left(F_{k}(z) \tilde{F}_{l}(z)\right) \downarrow_{2}=\delta(k-l), \quad k, l=0,1,
$$

which can be rewritten as

$$
F_{k}(z) \tilde{F}_{l}(z)+F_{k}(-z) \tilde{F}_{l}(-z)=2 \delta(k-l) .
$$

This can be shown [11] to be exactly equivalent to the condition that the filters $F_{0}(z)$ and $F_{1}(z)$ form a PU-pair. When $k=l$, we refer to (2.13) as the unit-energy condition. Now consider a general $L$-level tree, drawn in terms of the polyphase matrix of the filters on all levels. Fig. 4
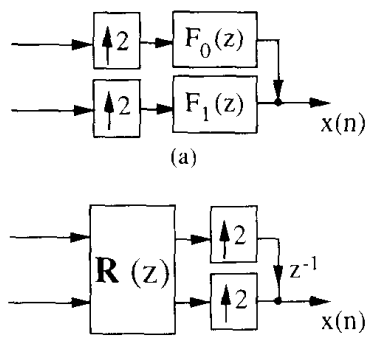

(b)

Fig. 3. (a) A two channel filter bank. (b) A two channel bank drawn in terms of the polyphase matrix.

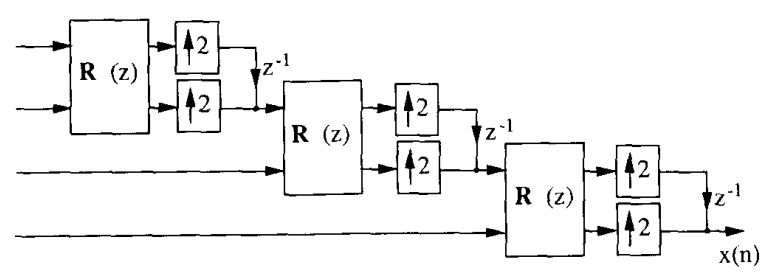

Fig. 4. A tree-structured filter bank drawn in terms of the polyphase matrices.

shows the case $L=3$. Let the polyphase matrix be paraunitary, i.e., it satisfies (1.3). Then, it has been shown in [16] that the wavelet basis generated by that tree is orthonormal.

Orthonormality of basis functions is often a very desirable property in several applications.

\section{Some Results on Paraunitary Systems}

In this section we present a few basic results pertaining to paraunitary systems. Some of them are straightforward, but many are fundamental. All are included here for the sake of completeness.

Lemma 1: Let $A(z)$ be a FIR transfer function such that $(A(z)) \downarrow_{M}=1$, and let $A(z)$ have a factor of the form $c\left(z^{M}\right)$. Then $c(z)=k z^{l}$, for some constant $k$ and integer $l$. In other words, the $M$ polyphase components of $A(z)$ cannot have a common factor other than of the form $\mathrm{kz}^{\prime}$.

Proof: Let

$$
A(z)=c\left(z^{M}\right) a(z) .
$$

Hence, $\left(c\left(z^{M}\right) a(z)\right) \downarrow_{M}=1$. Using the noble identity [11], we get

$$
c(z)\left[(a(z)) \downarrow_{M}\right]=1 .
$$

Since this is a product of two FIR functions, matching zeros on both sides of the above equation, we get $c(z)=$ $k z^{l}$.

Now, $A(z)$ can be written in terms of its polyphase components as in (1.1). If the $a_{i}(z) i=0, \cdots, M-1$ had a common factor, $A(z)$ could be written as in (3.1), which we have shown is not possible unless the common factor is of the form $k z^{\prime}$. Hence the $M$ polyphase components of a function $A(z)$ satisfying $(A(z)) \downarrow_{M}=1$ cannot have a common factor other than of the restricted form. 
Lemma 2: Given a FIR transfer-function $A(z)$ satisfying

$$
(A(z) \tilde{A}(z)) \downarrow_{2}=1
$$

we can always find a function $B(z)$ such that $A(z)$ and $B(z)$ form a PU-pair.

Proof: Since $A(z)$ is FIR, it can be multiplied by $z^{m}$ (for some $m$ positive or negative), so that $C(z)=z^{m} A(z)$ is causal with $c(0) \neq 0$. Since $A(z)$ satisfies $(3.3), C(z)$ also satisfies (3.3). Hence from [11], we know that the degree $N$ of $C(z)$ is constrained to be odd. Choose

$$
D(z)=z^{-N} \tilde{C}(-z)
$$

and let $B(z)=z^{-m} D(z)$. If $A(z)=a_{0}\left(z^{2}\right)+z^{-1} a_{1}\left(z^{2}\right)$ and $B(z)=b_{0}\left(z^{2}\right)+z^{-1} b_{1}\left(z^{2}\right)$, it can be verified that the above choice of $B(z)$ implies the relations

$$
\begin{aligned}
& b_{0}(z)=z^{-(N-1) / 2} \tilde{a}_{1}(z) \\
& b_{1}(z)=-z^{-(N-1) / 2} \tilde{a}_{0}(z) .
\end{aligned}
$$

It can be shown by direct substitution that with this choice, the filters $A(z)$ and $B(z)$ form a PU-pair.

Note that condition (3.3) means that $(A(z) \tilde{A}(z))$ is a halfband filter [11]. Equivalently, $A(z)$ is a spectral factor of a halfband filter.

Lemma 3: Given a FIR transfer-function $A(z)$ satisfying (3.3), and a function $P(z)$ satisfying

$$
(A(z) \tilde{P}(z)) \downarrow_{2}=0
$$

$P(z)$ can always be written as

$$
P(z)=p\left(z^{2}\right) B(z)
$$

where $B(z)$ is as defined in Lemma 2 .

Proof: The functions can be written in terms of their polyphase components as

$$
\begin{aligned}
& A(z)=a_{0}\left(z^{2}\right)+z^{-1} a_{1}\left(z^{2}\right) \\
& P(z)=p_{0}\left(z^{2}\right)+z^{-1} p_{1}\left(z^{2}\right) .
\end{aligned}
$$

Equation (3.7) can be written in terms of the polyphase components as

$$
a_{0}(z) \tilde{p}_{0}(z)+a_{1}(z) \tilde{p}_{1}(z)=0 .
$$

Note that by Lemma 1 , if $a_{0}(z)$ and $a_{1}(z)$ have a common factor, it is of the form $k z^{l}$. Thus in order that (3.11) be satisfied, we need

$$
\begin{aligned}
& p_{0}(z)=p(z) \tilde{a}_{1}(z) z^{-l} \\
& p_{1}(z)=-p(z) \tilde{a}_{0}(z) z^{-l}
\end{aligned}
$$

which means $P(z)$ can be written as in (3.8).

Lemma 4: If $A_{i}(z), i=0, \cdots, M-1$ form a PUset, then they cannot have a common factor except of the form $k z^{\prime}$.

Proof: Since $A_{i}(z), i=0, \cdots, M-1$ form a PUset, they satisfy [11]

$$
\sum_{i=0}^{M-1} A_{i}(z) \tilde{A}_{i}(z)=M \quad \text { for all } z
$$

If $A_{i}(z), i=0, \cdots, M-1$ have common factor $(z-$ $z_{0}$ ) with $z_{0} \neq 0$, the left-hand side would vanish at $z=$ $z_{0}$, violating (3.14).

Lemma 5: Let the filters $G_{k}(z), k=0,1,2, \cdots, M$ - 1 form a PU-set. Then,

$$
\left(A(z) G_{k}(z)\right) \downarrow_{M}=0, \quad \text { for } k=0,1,2, \cdots, M-1
$$

implies $A(z) \equiv 0$.

Proof: Consider Fig. 5(a) redrawn as Fig. 5(b). $\boldsymbol{E}(z)$ is the type I polyphase matrix of the filters $G_{k}(z), k=0$, $1,2, \cdots, M-1$. Apply an impulse $\delta(n)$ as an input to the system. Equation (3.15) means that the output of the system $\boldsymbol{y}(z) \equiv \mathbf{0}$. Since the matrix $\boldsymbol{E}(z)$ is paraunitary, this means that $v(z) \equiv \mathbf{0}$. Hence the output of the filter $A(z)$ in response to the impulse $\delta(n)$ is zero, i.e., $A(z) \equiv$ 0.

Lemma 6: Let the filters $G_{k}(z), k=0,1,2, \cdots, M$ - 1 form a PU-set. Let $a$ and $b$ be relatively prime integers, and let $b$ be a factor of $M$. If

$$
\left(A(z) G_{k}\left(z^{a}\right)\right) \downarrow_{b}=0, \quad k=0,1,2, \cdots, M-1
$$

then $A(z) \equiv 0$.

Proof: In Fig. 5(a), imagine that each $G_{k}(z)$ is replaced with $G_{k}\left(z^{a}\right)$, and the decimation factor is made $b$. This can be redrawn in terms of the polyphase matrix as in Fig. 6. Again, apply an impulse $\delta(n)$ as an input to the system. Equation (3.16) means that the output of the system $\boldsymbol{y}(z) \equiv \mathbf{0}$. Since the matrix $\boldsymbol{E}(z)$, which is the polyphase matrix of the filters $G_{k}(z)$ is paraunitary, the matrix $\boldsymbol{E}\left(z^{a M / b}\right)$ is also paraunitary. This means that $\boldsymbol{v}(z) \equiv \mathbf{0}$. Now, since $a$ and $b$ are relatively prime, it can be shown that the output of the filter $A(z)$ is zero, hence proving the Lemma.

Lemma 7: Let $A(z)$ be some rational transfer function, and let $L$ be any integer. Then, there exists a $C(z)$ such that

$$
(A(z) \tilde{A}(z)) \downarrow_{L}=C(z) \tilde{C}(z)
$$

Furthermore, if $A(z)$ is FIR, $C(z)$ is also FIR.

Proof: Observe that $A\left(e^{j \omega}\right) A^{*}\left(e^{j \omega}\right) \geq 0$, and so its any $L$-fold decimated version. Hence we can rewrite it as $C\left(e^{j \omega}\right) C^{*}\left(e^{j \omega}\right)$. By analytic continuation, we have (3.17).

If $A(z)$ is FIR, its $L$-fold decimated version is FIR, and so $C(z)$ is also FIR.

\section{Orthonormal Wavelets and Binary Tree- Structured Filter Banks}

In this section, we study further the connection between orthonormality of wavelet bases and paraunitariness of matrices in a binary tree-structured filter bank. All wavelet bases we consider are of finite duration, or FIR, unless stated otherwise. Finite duration wavelets have been referred to as "compactly supported wavelets" in [10]. Consider a $L$-level binary tree-structured synthesis filter bank used traditionally for generating a wavelet basis, 


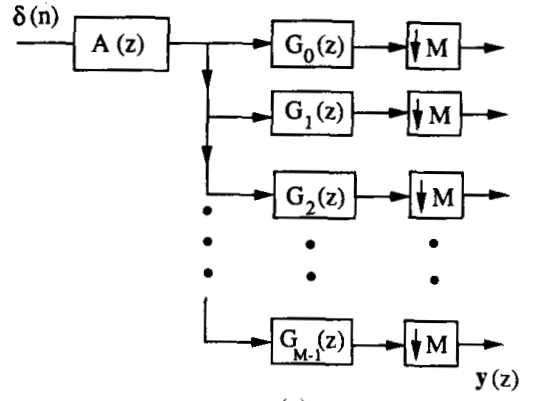

(a)

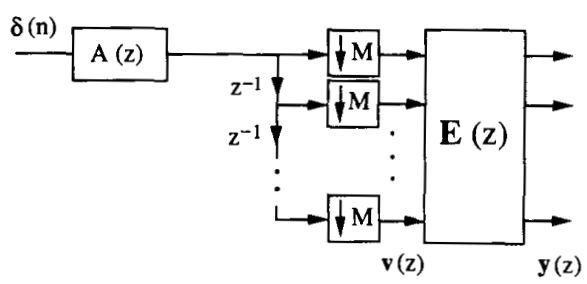

(b)

Fig. 5. Demonstration for Lemma 5. (a) The $M$ channel filter bank. (b) The filter bank in terms of the polyphase matrix.

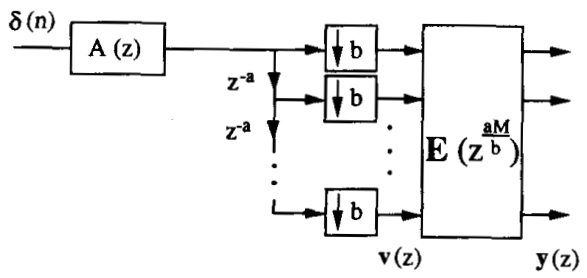

Fig. 6. Demonstration for Lemma 6.

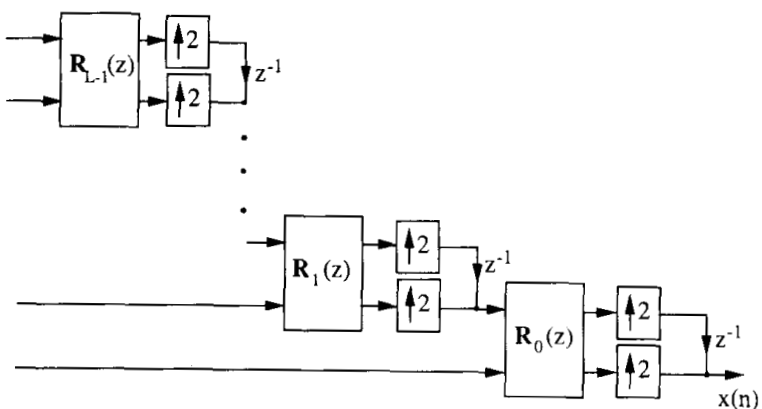

Fig. 7. A binary tree-structured synthesis bank in terms of the polyphase matrices.

drawn in terms of the polyphase matrices of the filters. Fig. 4 is an example for $L=3$. It is known [16] that if the matrix $\boldsymbol{R}(z)$ is paraunitary, i.e., it satisfies (1.3), the wavelet basis generated by this tree-structure is orthonormal.

First, we shall consider a simple generalization of Fig. 4. Consider Fig. 7. This is also a binary tree-structured synthesis filter bank, but the filters (and hence their polyphase matrix) on each level are different. We now prove:
Theorem 1: Consider a $L$-level binary tree-structured filter bank. Let the polyphase matrices on each level, $\boldsymbol{R}_{i}(z)$ $i=0, \cdots, L-1$ be paraunitary. Then, the wavelet basis generated by this tree is orthonormal.

Proof: The proof of this result is a straightforward generalization of the one given in [16], for tree structures having the same paraunitary matrix on each level. We present it here for the sake of completeness.

We prove this result by induction. Consider a $L$-level tree (Fig. 8(a)) drawn as a traditional synthesis filter bank (Fig. 8(b)). The filters $F_{k}(z)$ are given by the relations

$$
\begin{aligned}
& F_{0}(z)=H_{s_{0}}(z) \\
& F_{k}(z)=H_{s_{k}}\left(z^{2^{k}}\right) \prod_{i=0}^{k-1} G_{s_{i}}\left(z^{2^{i}}\right) \quad k=1, \cdots, L-1 \\
& F_{L}(z)=G_{s_{L}, 1}\left(z^{2^{L-1}}\right) \prod_{i=0}^{L-2} G_{s_{i}}\left(z^{2^{2}}\right)
\end{aligned}
$$

The tree has $L+1$ branches. Fig. 9(a) shows two of these branches, with $k \geq l$. Suppose we add another level to the tree. This adds a new branch, and modifies the existing branches as shown in Fig. 9(b). Assuming that a) the wavelet basis is orthonormal for the $L$-level tree, and that b) the new set of filters $\left(G_{s L}(z), H_{s L}(z)\right)$ has a polyphase matrix which is paraunitary, we prove that the waveletbasis generated by the $(L+1)$-level tree is also orthonormal. From the paraunitariness of their polyphase matrix, we know that

$$
\begin{gathered}
\left(G_{s L}(z) \tilde{G}_{s_{L}}(z)\right) \downarrow_{2}=1 \quad\left(H_{s_{L}}(z) \tilde{H}_{s_{L}}(z)\right) \downarrow_{2}=1 \\
\left(G_{s_{L}}(z) \tilde{H}_{s_{L}}(z)\right) \downarrow_{2}=0 .
\end{gathered}
$$

Orthonormality of the $L$-level tree implies

$$
\left(F_{k}(z) \tilde{F}_{l}(z)\right) \downarrow_{2^{l+1}}=\delta(k-l), \quad 0 \leq l \leq k \leq L-1 .
$$

The three branches of the $L+1$-level tree shown in Fig. 9(b) can be redrawn as in Fig. 9(c), where

$$
S_{k}(z) \equiv F_{k}\left(z^{2}\right) G_{s L}(z), \quad S_{l}(z) \equiv F_{l}\left(z^{2}\right) G_{s L}(z) .
$$

Thus,

$$
\left(S_{k}(z) \tilde{S}_{l}(z)\right) \downarrow_{2^{i+2}}=\left(F_{k}\left(z^{2}\right) \tilde{F}_{l}\left(z^{2}\right) G_{s L}(z) \tilde{G}_{s L}(z)\right) \downarrow_{2^{i+2}}
$$

$$
\begin{aligned}
& =\left(F_{k}(z) \tilde{F}_{l}(z)\left(G_{s L}(z) \tilde{G}_{s L}(z)\right) \downarrow_{2}\right) \downarrow_{2^{i+1}} \\
& =\delta(k-l) .
\end{aligned}
$$

We have used (4.4) and (4.5) to arrive at the first answer. Also,

$$
\begin{aligned}
\left(S_{k}(z) \tilde{H}_{s_{L}}(z)\right) \downarrow_{2} & =F_{k}(z)\left(G_{s_{L}}(z) \tilde{H}_{s_{L}}(z)\right) \downarrow_{2} \\
& =0
\end{aligned}
$$

using (4.4). This is sufficient to prove that the wavelet basis generated by the $L+1$-level tree is orthonormal. 


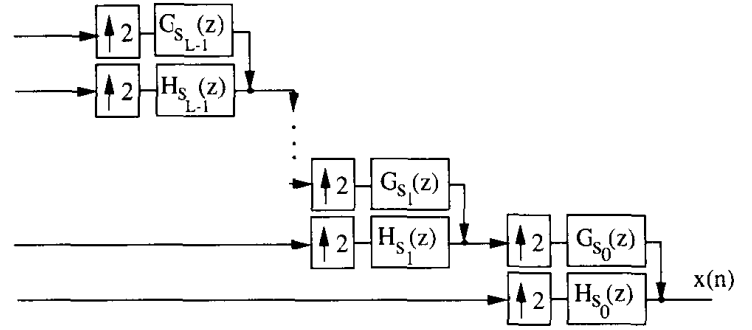

(a)
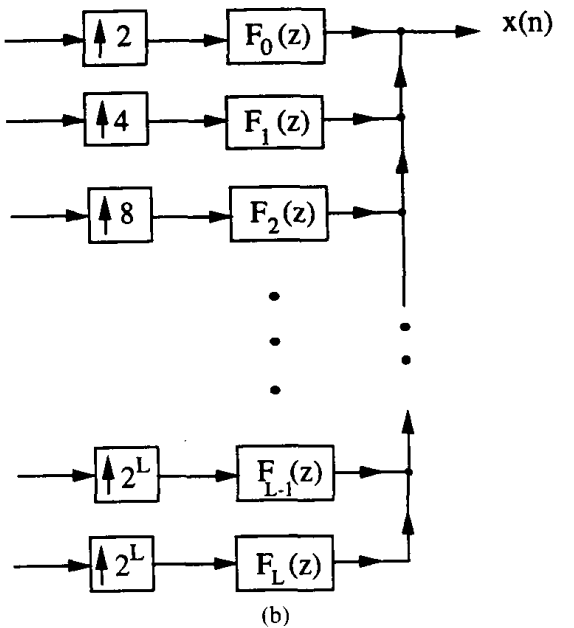

Fig. 8. (a) A binary tree-structured synthesis bank. (b) A binary tree-structure redrawn as a traditional filter bank.

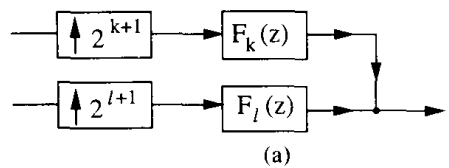

(a)

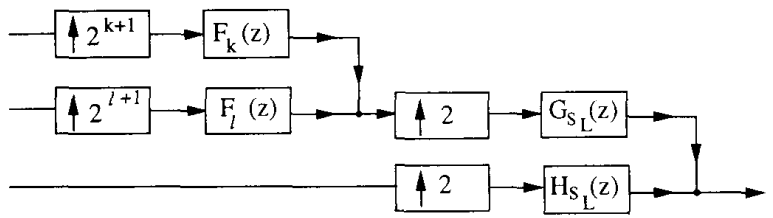

(b)

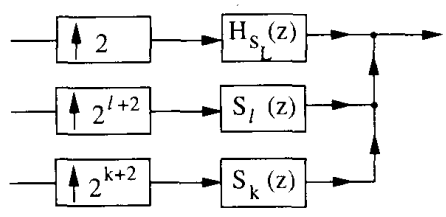

(c)

Fig. 9. (a) Two branches of a $L$-level tree. (b) Filters on the $(L+1)$ level are added. (c) Tree resulting from the addition of one more level

We shall now consider the converse of the above question, namely, is it possible to generate all orthonormal wavelet bases using binary tree-structured filter banks? It turns out that this is true in the case of finite duration discrete-time orthonormal wavelets.
Theorem 2: Every finite duration discrete-time orthonormal wavelet basis can be generated by a binary treestructured filter bank having paraunitary matrices on all levels.

Proof: Let $y_{k}(m)$ denote the wavelet coefficients at resolution $k$, and let $\eta_{k m}(n)$ denote the wavelet basis functions. We recall that the original signal $x(n)$ can be reconstructed from its wavelet coefficients as in (2.3). We also recall that in a tree-structured filter bank, the synthesis filters are related to the wavelet basis functions as in (2.4) and (2.5). Consider a binary tree-structured synthesis bank, drawn as a conventional filter bank in Fig. 8(b). Notice the increasing interpolation ratios. Since we are dealing with compactly supported bases, the filters $F_{k}(z)$ are FIR. We shall also assume that they are causal with $f_{k}(0) \neq 0$. This assumption is not restrictive, since we are dealing with FIR functions, and any FIR function can be brought into this form by a suitable advance/delay operation. Orthonormality of the wavelet basis implies (2.7) to $(2.15)$. For the sake of convenience, we reproduce below the orthonormality condition in the $z$-domain,

$$
\begin{aligned}
&\left(F_{k}(z) \tilde{F}_{l}(z)\right) \downarrow_{2^{l+1}}=\delta(k-l), \quad 0 \leq k \leq l \leq L-1 \\
&\left(F_{L}(z) \tilde{F}_{l}(z)\right) \downarrow_{2^{l+1}}=0 \quad l=0, \cdots, L-1 \\
&\left(F_{L}(z) \tilde{F}_{L}(z)\right) \downarrow_{2^{l}}=1 .
\end{aligned}
$$

Our task is to show that a set of functions $F_{i}(z)$ satisfying the above condition can always be generated using a tree-structured filter-bank having paraunitary matrices on all levels. In other words, given the filters $F_{k}(z)$ in Fig. 8(b), we want to obtain filters $\left(G_{s_{i}}(z), H_{s_{i}}(z)\right)$ in Fig. 8(a) such that they form PU-pairs for all $i$. We now give a constructive proof showing that this is always possible.

Choose

$$
H_{s 0}(z)=F_{0}(z) .
$$

Hence from (2.13),

$$
\left(H_{s 0}(z) \tilde{H}_{s 0}(z)\right) \downarrow_{2}=1 .
$$

Choose

$$
G_{s 0}(z)=z^{-N_{0}} \tilde{H}_{s_{0}}(-z)
$$

where $N_{0}$ is the degree of $H_{s 0}(z)$. Note that $G_{s 0}(z)$ and $H_{s 0}(z)$ are both FIR, and $g_{s 0}(0) \neq 0, h_{s 0}(0) \neq 0$. Lemma 2 assures us that $G_{s 0}(z)$ and $H_{s 0}(z)$ form a PU-pair.

Now from (2.13) and (2.14),

$$
\left(F_{j}(z) \tilde{F}_{0}(z)\right) \downarrow_{2}=0 \quad \text { for } j=1,2, \cdots, L .
$$

Hence by Lemma 3 , the $F_{j}(z)$ are expressible as

$$
F_{j}(z)=f_{j}^{\prime}\left(z^{2}\right) G_{s o}(z)
$$

where the $f_{j}^{\prime}(z)$ are FIR and do not have a zero at infinity.

Now in particular, $F_{1}(z)$ is expressible as

$$
F_{1}(z)=f_{1}^{\prime}\left(z^{2}\right) G_{s i}(z)
$$

Choose

$$
H_{s_{1}}(z)=f_{1}^{\prime}(z)
$$


From (2.13) we know that

$$
\left(F_{1}(z) \tilde{F}_{1}(z)\right) \downarrow_{4}=1
$$

therefore,

$$
\left(H_{s_{1}}\left(z^{2}\right) G_{s 0}(z) \tilde{H}_{s 1}\left(z^{2}\right) \tilde{G}_{s 0}(z)\right) \downarrow_{4}=1 .
$$

Using noble identity, this becomes

$$
\left[H_{s 1}(z) \tilde{H}_{s 1}(z)\left(G_{s 0}(z) \tilde{G}_{s 0}(z)\right) \downarrow_{2}\right] \downarrow_{2}=1
$$

and hence, from the fact that $G_{s 0}(z)$ belongs to a PU-pair,

$$
\left(H_{s_{1}}(z) \tilde{H}_{s_{1}}(z)\right) \downarrow_{2}=1 \text {. }
$$

Choose

$$
G_{s_{1}}(z)=z^{-N_{1}} \tilde{H}_{s_{1}}(-z)
$$

where $N_{1}$ is the degree of $H_{s_{1}}(z)$. Hence $G_{s_{1}}(z)$ and $H_{s_{1}}(z)$ form a PU-pair, and both are FIR with $g_{s 1}(0) \neq 0$ and $h_{s 1}(0) \neq 0$.

Now from (2.13),

$$
\left(F_{j}(z) \tilde{F}_{1}(z)\right) \downarrow_{4}=0 \quad \text { for } j=2, \cdots, L .
$$

Using (4.15) and (4.17) this becomes

$$
\left[f_{j}^{\prime}\left(z^{2}\right) G_{s 0}(z) \tilde{H}_{s 1}\left(z^{2}\right) \tilde{G}_{s 0}(z)\right] \downarrow_{4}=0
$$

which simplifies to

$$
\left(f_{j}^{\prime}(z) \tilde{H}_{s_{1}}(z)\right) \downarrow_{2}=0 \quad j=2,3, \cdots, L .
$$

Hence by Lemma 3 , the $f_{j}^{\prime}(z)$ are expressible as

$$
f_{j}^{\prime}(z)=f_{j}^{\prime \prime}\left(z^{2}\right) G_{s_{1}}(z) \quad j=2,3, \cdots, L
$$

where the filters $f_{j}^{\prime \prime}(z)$ are FIR and do not have a zero at infinity. Hence using (4.15), we get

$$
F_{j}(z)=f_{j}^{\prime \prime}\left(z^{4}\right) G_{s !}\left(z^{2}\right) G_{0}(z) \quad j=2, \cdots, L .
$$

Now in particular, $F_{2}(z)$ is expressible as

$$
F_{2}(z)=f_{2}^{\prime \prime}\left(z^{4}\right) G_{s_{1}}\left(z^{2}\right) G_{s 0}(z)
$$

with $f_{2}^{\prime \prime}(z)$ being FIR. Hence choose

$$
H_{s 2}(z)=f_{2}^{\prime \prime}(z) \text {. }
$$

Therefore,

$$
F_{2}(z)=H_{s 2}\left(z^{4}\right) G_{s 1}\left(z^{2}\right) G_{s 0}(z) .
$$

In general, since $F_{k}(z)$ is orthogonal to $F_{i}(z)$, for $i=$ $0,1,2, \cdots, k-1$, it can be expressed as

$$
F_{k}(z)=H_{s k}\left(z^{2 k}\right) G_{s k-1}\left(z^{2^{k-1}}\right) \cdots G_{s 0}(z)
$$

and from the unit-energy property $(2.13)$ we have

$$
\left(H_{s k}(z) \tilde{H}_{s k}(z)\right) \downarrow_{2}=1 \text {. }
$$

At every stage, the filters $G_{s k}(z)$ are chosen such that

$$
G_{s_{k}}(z)=z^{-N_{k}} \tilde{H}_{s_{k}}(-z)
$$

where $N_{k}$ is the degree of $H_{s k}(z)$. Hence the filters $\left(G_{s_{i}}(z)\right.$, $\left.H_{s_{i}}(z)\right)$ form PU-sets on all levels, They are all FIR, causal, and do not have a zero at infinity.

In particular, on the final level we have

$$
F_{L-1}(z)=H_{s_{L-1}}\left(z^{2^{L-1}}\right) G_{s_{L-2}}\left(z^{2 L-2}\right) \cdots G_{s 0}(z)
$$

with

$$
\left(H_{s_{L-1}}(z) \tilde{H}_{s_{L-1}}(z)\right) \downarrow_{2}=1 .
$$

Since the function $F_{L}(z)$ is orthogonal to $F_{i}(z)$, for $i=0$, $1,2, \cdots, L-2$, following the general procedure, it can be expressed as

$$
F_{L}(z)=g\left(z^{2^{L-1}}\right) G_{s_{L-2}}\left(z^{2^{L-2}}\right) \cdots G_{s_{0}}(z)
$$

where $g(z)$ is FIR. Let $G_{s_{L-1}}(z)=z^{-N_{L-1}} \tilde{H}_{s_{L-1}}(-z)$. Since $F_{L}(z)$ is also orthonormal to $F_{L-1}(z)$, by Lemma 3 , $g(z)=g^{\prime}\left(z^{2}\right) G_{s L-1}(z)$. It can be verified that the unit-energy condition (2.16) applied to $F_{L}(z)$ implies $g^{\prime}(z)=1$. Thus,

$$
F_{L}(z)=G_{s L-1}\left(z^{2^{L-1}}\right) G_{s L-1}\left(z^{2^{L-2}}\right) \cdots G_{s 0}(z)
$$

with $G_{s_{L-1}}(z)$ and $H_{s L-1}(z)$ forming a PU-pair.

Thus, we have shown that given any finite duration discrete-time orthonormal basis $F_{i}(z)$, for $i=0,1,2, \cdots$, $L$, it is always possible to generate it using a tree-structured filter bank having paraunitary matrices on all levels; i.e., Fig. 8(b) can always be redrawn as Fig. 8(a), with the filters $\left(G_{s_{i}}(z), H_{s_{i}}(z)\right)$ forming PU-pairs.

Now consider Fig. 8(a). We know that $G_{s i}(z)$ belongs to a PU-pair, is causal, and $g_{s_{i}}(0) \neq 0$. Hence it cannot have a factor of the form $c\left(z^{2}\right)$, other than a constant. Thus no factor (except a constant) of $G_{s_{i}}(z)$ can be moved left across the interpolators. Also, since the filters $\left(G_{s_{i}}(z)\right.$, $\left.H_{s_{i}}(z)\right)$ on each level form a PU-pair, are causal, FIR, and without a zero at infinity, by Lemma 4 , they cannot have a common factor other than a constant. Hence no factor (except a constant) common to these two can be moved right across the interpolators. This gives the following corollary to the above theorem.

Corollary 1: If a finite duration orthonormal wavelet basis is generated using a tree-structured filter bank, the polyphase matrices of the filters on each level have to satisfy the condition

$$
\tilde{\boldsymbol{E}}_{i}(z) \boldsymbol{E}_{i}(z)=c_{i} \boldsymbol{I} \quad \text { for all } z, \quad i=0, \cdots, L-1 .
$$

From the above theorem, we have another corollary regarding the linearity of phase of the wavelet basis.

Corollary 2: If a orthonormal wavelet basis has filters with linear phase, the filters $G_{s_{i}}(z)$ and $H_{s_{i}}(z)$ on each level have the form $c_{1} z^{-l_{1}}+c_{2} z^{-l_{2}}$ for some constants $c_{1}$ and $c_{2}$ and integers $l_{1}$ and $l_{2}$.

To see this, note that orthonormality of the wavelet basis implies that the polyphase matrices on each level have to satisfy (4.38). In particular, since $F_{0}(z)$ has linear phase, we know from [14] that $H_{s 0}(z)=c_{1} z^{-l_{1}}+c_{2} z^{-t_{2}}$. Since $G_{s 0}(z)$ and $H_{s 0}(z)$ form a PU-set, $G_{s 0}(z)$ can also be written in such a form. Now, since $F_{1}(z)$ and $G_{s 0}(z)$ both have linear phase, $H_{s 1}(z)$ also has linear phase, and it is therefore restricted to the form stated above. Therefore, $G_{s_{0}}(z)$ also has this form. Continuing such an argument down the tree, we see that each of the filters $G_{s_{i}}(z)$ and $H_{s_{i}}(z)$ have the form $c_{1} z^{-l_{1}}+c_{2} z^{-l_{2}}$. 


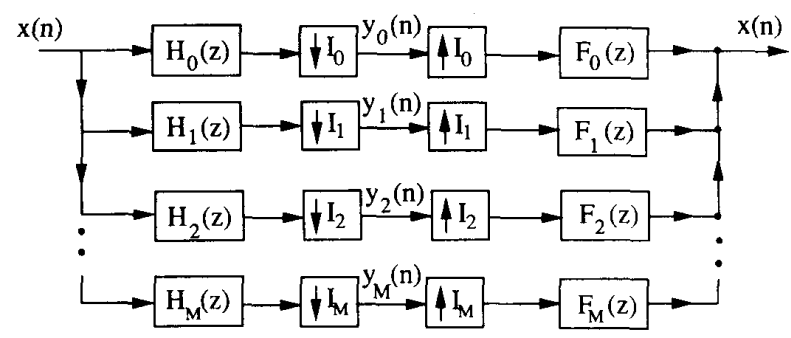

Fig. 10. A nonuniform filter bank.

\section{Orthonormality of Wavelet Packets}

The basis functions of a filter bank with nonuniform decimation ratios are called wavelet packets [1], [17]. Fig. 10 shows a schematic of such a filter bank. If $y_{k}(n)$ are the inputs to the synthesis filters, then (assuming that this is perfect reconstruction system) we can write

$$
x(n)=\sum_{k=0}^{M} \sum_{m} y_{k}(m) f_{k}\left(n-I_{k} m\right)
$$

where $I_{k}$ is the interpolator preceding $F_{k}(z)$, and $M+1$ is the total number of filters. From the similarity of the above equation with (2.3), we refer to the quantities $y_{k}(n)$ as the generalized wavelet coefficients. The set of functions $f_{k}$ (n - $I_{k} m$ ) is the generalized wavelet basis, or wavelet packets. Analogous to (2.6), we say the basis functions are orthonormal if they satisfy the relations

$$
\sum_{n=-\infty}^{\infty} f_{k}\left(n-I_{k} m\right) f_{l}^{*}\left(n-I_{l} i\right)=\delta(k-l) \delta(m-i) .
$$

With a change of variables this can be rewritten as (see the Appendix)

$$
\sum_{n=-\infty}^{\infty} f_{k}(n) f_{l}^{*}\left(n-g_{l k} i\right)=\delta(k-l) \delta(i)
$$

where $g_{l k}$ is the $\operatorname{gcd}$ of $\left(I_{k}, I_{l}\right)$. In the $z$-domain, this becomes

$$
\left(F_{k}(z) \tilde{F}_{l}(z)\right) \downarrow_{g l k}=\delta(k-l) .
$$

\section{Generalized Tree-Structures and ORTHONORMAL BASES}

In Section III we saw that wavelet bases could be generated using a binary tree-structured QMF filter bank. The wavelet basis functions were seen to be orthonormal if the filters on each level of the tree had a polyphase matrix which was paraunitary, and conversely. Fig. 1(b) shows a binary tree-structured filter bank traditionally used for generating wavelet bases. Now consider a general treestructured filter bank. Fig. 11(a) shows one such example of the synthesis bank. This is associated with a corresponding analysis bank not shown in the figure.

Consider the binary tree-structured synthesis bank (Fig. 1(b)), and a general tree-structured synthesis bank (Fig. 11(a)). Comparing the two, we note two important differ-

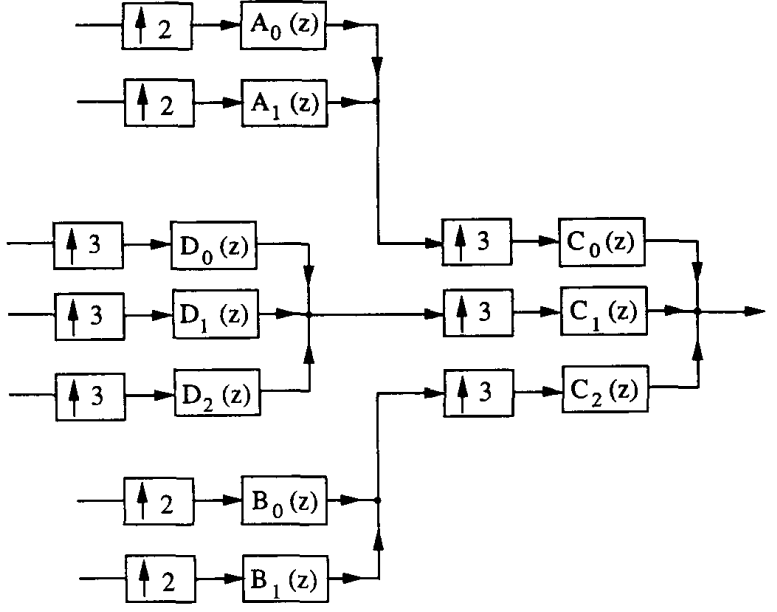

(a)
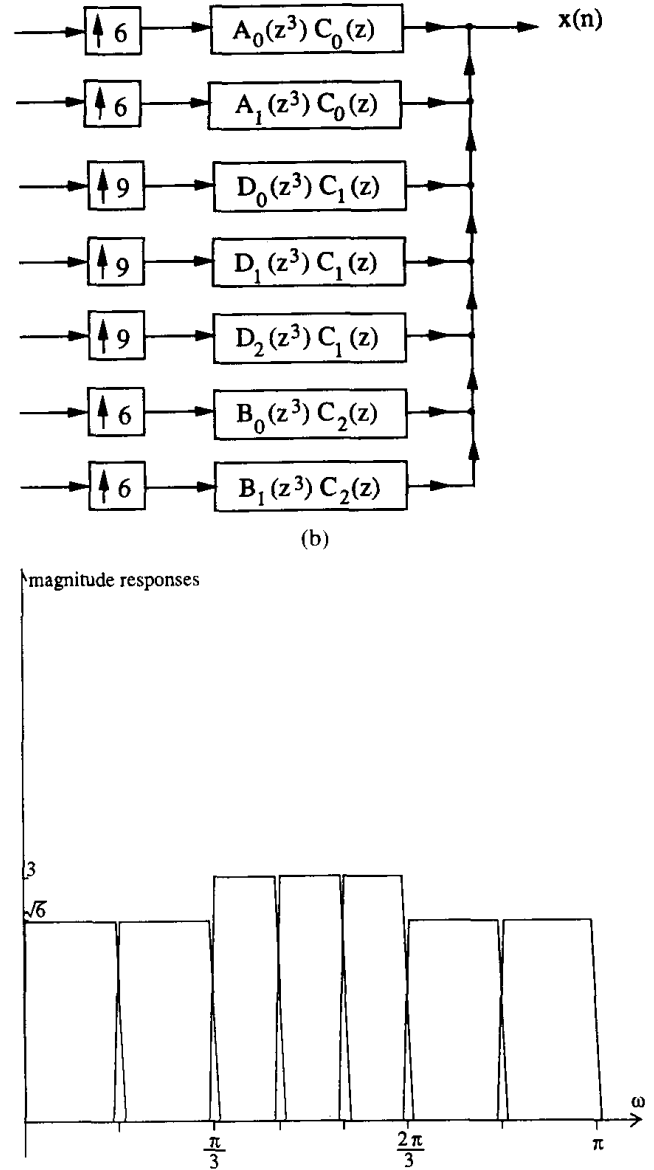

(c)

Fig. 11. (a) A generalized tree-structured synthesis bank. (b) A generalized tree drawn as a traditional synthesis filter bank. (c) Typical responses of filters produced by a generalized tree.

ences. First, (going right to left) we see that in a general tree, any branch on a certain level can divide further, whereas in a binary tree, only one of the two branches on 
any level branches out further. Second, for a generalized tree, each level may have different number of filters, in contrast to a binary tree in which each level has exactly two filters. Thus, a maximally decimated filter bank in which one or more branches on any level split further into branches is called an "arbitrary tree structured" filter bank. In the context of generalized tree structures, we need to rigorously define what we mean by a "level." In a tree-structured filter bank, filters whose outputs go into a single adder are said to be on the same level. Consider for example, Fig. 11(a). This tree has four levels, namely, i) $\left(D_{0}(z), D_{1}(z), D_{2}(z)\right)$; ii) $\left(A_{0}(z), A_{1}(z)\right)$; iii) $\left(B_{0}(z)\right.$, $\left.B_{1}(z)\right)$; iv) $\left(C_{0}(z), C_{1}(z), C_{2}(z)\right)$. To see this, note that for example, the outputs of the filters $D_{0}(z), D_{1}(z)$ and $D_{2}(z)$ go into a single adder (denoted by a heavy dot), and hence they are on the same level. On the other hand, the outputs of the filters $D_{0}(z)$ and $B_{0}(z)$ do not go into the same adder, and thus they are said to be on different levels. The word "level" used in the case of arbitrary trees does not have the strict connotation of "depth" as it does in the English language, or as in the case of binary trees (Fig. 1). Consequently, for generalized trees the levels are not numbered as they are in the case of binary trees. We do define something called the "input level," however. If none of the branches in a certain level further divide into branches (while going right to left in a synthesis core tree), such a level is called an "input level." Note that there can exist more than one input level for a general tree, whereas a binary tree has a distinct input level. For example, Fig. 11(a) has three input levels, namely, i) $\left(D_{0}(z)\right.$, $\left.D_{1}(z), D_{2}(z)\right)$; ii) $\left(A_{0}(z), A_{1}(z)\right)$; iii) $\left(B_{0}(z), B_{1}(z)\right)$. Note that $\left(C_{0}(z), C_{1}(z), C_{2}(z)\right)$ is not an input level.

We can guarantee perfect reconstruction property for such filter banks by appropriately choosing filters on each level. The tree structure therefore gives rise to a set of wavelet packets. The generalized tree structure can be redrawn as a traditional filter bank as in Fig. 11(b). Fig. 11(c) shows typical appearances of frequency responses of such a tree structure. Taking a cue from traditional wavelet theory, we now ask the question: Is there a relationship between the paraunitariness of filters on each level of the generalized tree and orthonormality of the resulting basis? The answer to this is provided by the two theorems in this section.

In this section too, we are dealing with finite duration discrete functions.

Theorem 3: If an arbitrary tree-structured FIR filter bank, such as one in Fig. 11(a), has filters on each level forming PU-sets, then the functions $f_{k}\left(n-I_{k} m\right)$ generated by that tree form an orthonormal basis.

Proof: We prove this result by induction. We know from [16] that the result is true for a 1-level tree, i.e., we know that if a set of filters have a polyphase matrix which is paraunitary, the filters form an orthonormal basis. We now assume that the result holds for a $L$-level tree, and adding levels to the tree, we show that the functions generated by the new tree also form an orthonormal basis. Consider Fig. 12(a). The functions in both filter banks are
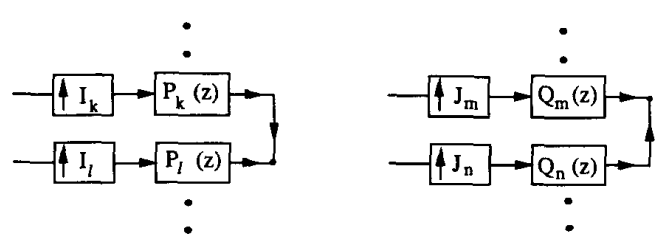

(a)

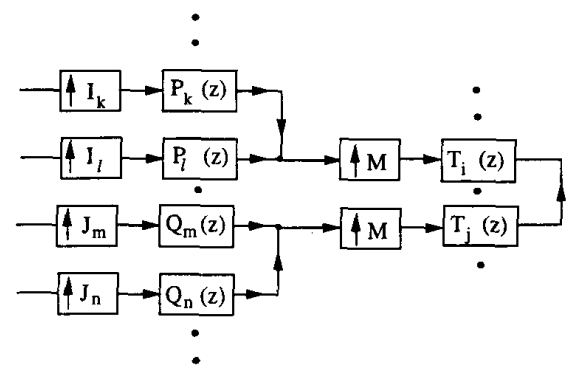

(b)

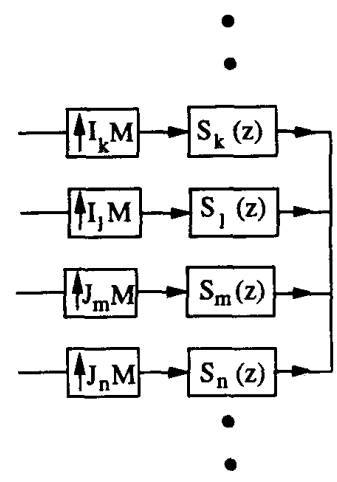

(c)

Fig. 12. (a) Two different filter banks. (b) The two filter banks in (a) are combined by adding one more level of filters. (c) Tree structure of (b) redrawn.

assumed to be orthonormal, i.e., they satisfy

$$
\begin{aligned}
& \left(P_{k}(z) \tilde{P}_{l}(z)\right) \downarrow_{g_{1}}=\delta(k-l), \quad g_{1}=\operatorname{gcd}\left(I_{k}, I_{l}\right) \\
& \left(Q_{m}(z) \tilde{Q}_{n}(z)\right) \downarrow_{g_{2}}=\delta(m-n), \quad g_{2}=\operatorname{gcd}\left(J_{m}, J_{n}\right) .
\end{aligned}
$$

We now combine these two by a common level to obtain the tree structure shown in Fig. 12(b). The $M$ filters on the new level added are paraunitary, i.e., they satisfy

$$
\left(T_{i}(z) \tilde{T}_{j}(z)\right) \downarrow_{M}=\delta(i-j) \quad i, j=0, \cdots, M-1 .
$$

This new tree can be redrawn again as in Fig. 12(c), where the filters are given as

$$
\begin{aligned}
& S_{k}(z)=P_{k}\left(z^{M}\right) T_{i}(z) \\
& S_{l}(z)=P_{l}\left(z^{M}\right) T_{i}(z)
\end{aligned}
$$




$$
\begin{aligned}
& S_{m}(z)=Q_{m}\left(z^{M}\right) T_{j}(z) \\
& S_{n}(z)=Q_{n}\left(z^{M}\right) T_{j}(z) .
\end{aligned}
$$

To prove orthogonality of the new basis, it is sufficient to show that

$$
\left(S_{k}(z) \tilde{S}_{k}(z)\right) \downarrow_{I_{k} M}=1
$$

and that

$$
\begin{gathered}
\left(S_{k}(z) \tilde{S}_{l}(z)\right) \downarrow_{g_{3}}=0, g_{3}=\operatorname{gcd}\left(I_{k} M, I_{l} M\right), \\
\left(S_{k}(z) \tilde{S}_{m}(z)\right) \downarrow_{g_{4}}=0, g_{4}=\operatorname{gcd}\left(I_{k} M, J_{m} M\right) .
\end{gathered}
$$

Consider,

$$
\left(S_{k}(z) \tilde{S}_{k}(z)\right) \downarrow_{l_{k} M}=\left(P_{k}\left(z^{M}\right) T_{i}(z) \tilde{P}_{k}\left(z^{M}\right) \tilde{T}_{i}(z)\right) \downarrow_{l_{k} M} .
$$

Using the noble identity, this becomes

$$
\begin{aligned}
\left(P_{k}(z) \tilde{P}_{k}(z)\left(T_{i}(z) \tilde{T}_{i}(z)\right) \downarrow_{M}\right) \downarrow_{I_{k}} & =\left(P_{k}(z) \tilde{P}_{k}(z)\right) \downarrow_{l_{k}} \\
& =1 .
\end{aligned}
$$

This proves $(6.8)$. The unit-energy property for the other transfer functions can be verified likewise.

Now, since $g_{3}=\operatorname{gcd}\left(I_{k} M, I_{l} M\right)$ and $g_{1}=\operatorname{gcd}\left(I_{k}, I_{l}\right)$, we have $g_{3}=M g_{1}$. Hence,

$$
\begin{aligned}
\left(S_{k}(z) \tilde{S}_{l}(z)\right) \downarrow_{g 3} & =\left(P_{k}\left(z^{M}\right) T_{i}(z) \tilde{P}_{l}\left(z^{M}\right) \tilde{T}_{i}(z)\right) \downarrow_{M_{g}} \\
& =\left(P_{k}(z) \tilde{P}_{l}(z)\left(T_{i}(z) \tilde{T}_{i}(z)\right) \downarrow_{M}\right) \downarrow_{g_{l}} \\
& =\left(P_{k}(z) \tilde{P}_{l}(z)\right) \downarrow_{g_{1}} \\
& =0
\end{aligned}
$$

using (6.1). This proves (6.9).

Now, $g_{4}=\operatorname{gcd}\left(J_{m} M, I_{k} M\right)$, so it is a multiple of $M$; let $g_{4}=a M$. Hence,

$$
\begin{aligned}
\left(S_{k}(z) \tilde{S}_{m}(z)\right) \downarrow_{g_{4}} & =\left(P_{k}\left(z^{M}\right) T_{i}(z) \tilde{Q}_{m}\left(z^{M}\right) \tilde{T}_{j}(z)\right) \downarrow_{a \cdot M} \\
& =\left(P_{k}(z) \tilde{Q}_{m}(z)\left(T_{i}(z) \tilde{T}_{j}(z)\right) \downarrow_{M}\right) \downarrow_{a} \\
& =\left(P_{k}(z) \tilde{Q}_{m}(z)(0)\right) \downarrow_{a} \\
& =0
\end{aligned}
$$

which proves $(6.10)$. Orthogonality of other pairs can similarly be verified.

Hence we have shown that the functions generated by the new tree also form an orthonormal basis. Since any tree-structured filter bank can be synthesized by this process of adding new levels, it proves our theorem.

We now turn our attention to the converse of this result. Unfortunately, the exact converse of the result in the previous theorem is not true. To see this, one only needs to consider a simple example of a tree-structured filter bank drawn in Fig. 11(a). This can be redrawn as in Fig. 11(b). Let the polyphase matrices of the filters in Fig. 11(a) be paraunitary. By the previous theorem, we know that the wavelet basis generated by this tree is orthonormal. Now

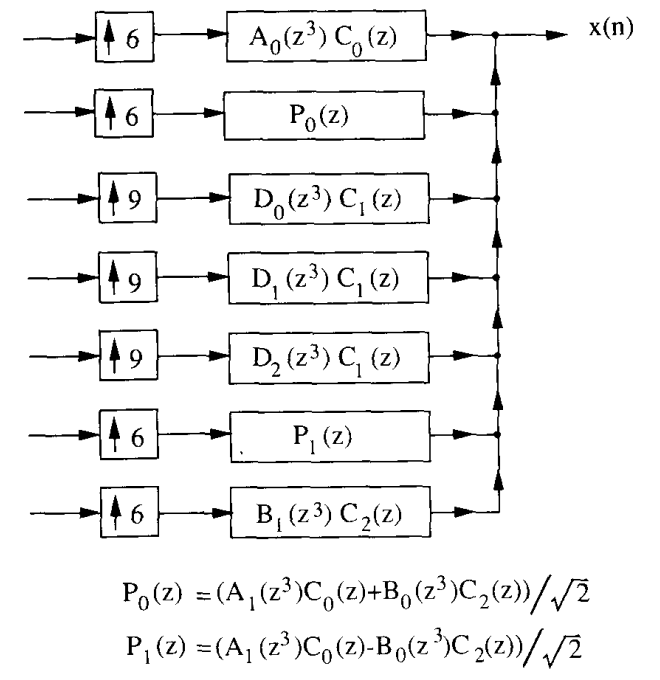

Fig. 13. A filter bank which cannot be generated by a tree structure

consider the filter bank shown in Fig. 13. $P_{0}(z)$ and $P_{1}(z)$ are the sum and difference of two filters in Fig. 11(b), as defined in the Fig. 13. Note that these filters form an orthonormal basis too, however, these cannot be generated using a tree structure. The reason is as follows. For this filter bank to be represented as a tree, we need that $P_{0}(z)$ be expressible as $A_{1}\left(z^{3}\right) C_{0}(z)$ or as $B_{0}\left(z^{3}\right) C_{2}(z)$ (compare with Fig. 11(b)). Neither is possible if $C_{0}(z) \neq C_{2}(z)$. But since $C_{0}(z)$ and $C_{2}(z)$ came from a PU-set to start with, the condition $C_{0}(z) \neq C_{2}(z)$ is guaranteed (by orthonormality). Thus, this filter bank cannot be generated using a tree structure.

We can, however prove the following weaker result.

Theorem 4: Let $F_{k}(z)$ be a set of FIR transfer functions satisfying (5.4). If they can be generated using a treestructured filter bank, they can be generated specifically by a tree having PU-sets on all levels.

Before proving this theorem we will prove the following two lemmas.

Lemma 8: Let $F_{k}(z)$ be a set of FIR basis functions which can be generated using a tree-structured filter bank, and let them satisfy the orthonormality condition (5.4). Then, the filters on an input level of the tree can be made to form a PU-set.

Proof: Given the FIR nature of the transfer functions involved, we shall assume without loss of generality that they are all causal. Consider the filter bank shown in Fig. 14(a). The filters $Q_{i}(z), i=0, \cdots, M-1$ are the filters on an input level of the tree. This can be redrawn as in Fig. 14(b). We are told that the functions $F_{m}(z)$ form an orthonormal basis, i.e., they satisfy (5.4). We are to show that it is possible to choose a set of filters $Q_{i}^{\prime}(z)$ in Fig. 14(c) for the input level such they form a PU-set.

Now, by orthonormality,

$$
\begin{aligned}
& \left(F_{k}(z) \tilde{F}_{l}(z)\right) \downarrow_{g 6}=\delta(k-l), \\
& \quad g_{6}=b M \quad k, l=0, \cdots, M-1
\end{aligned}
$$




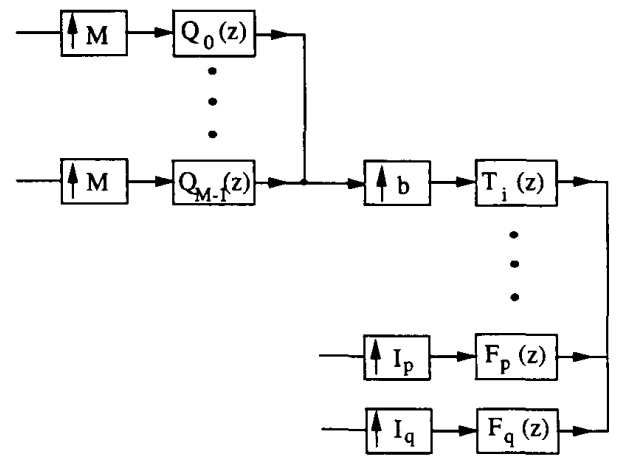

(a)
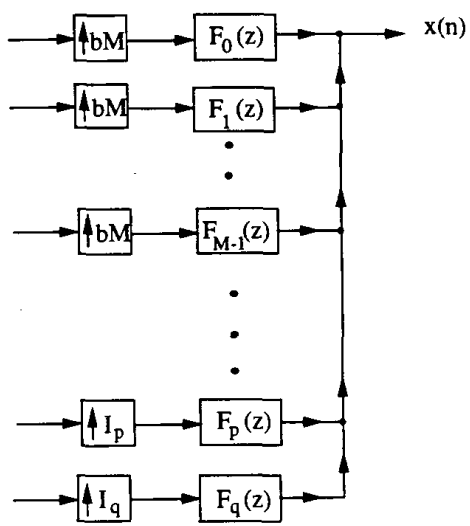

(b)

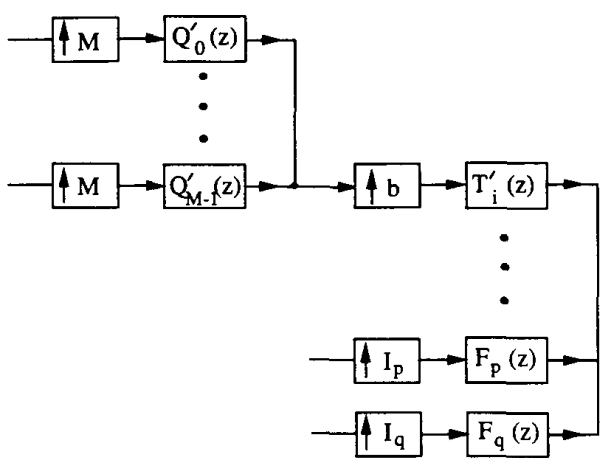

(c)

Fig. 14. (a) A filter bank showing only one input level. (b) The filter bank in (a) redrawn as a traditional filter bank. (c) A filter bank showing only one input level with modified filters.

where

$$
F_{k}(z)=Q_{k}\left(z^{b}\right) T_{i}(z), \quad k=0, \cdots, M-1
$$

Hence (6.22) means

$$
\begin{aligned}
& \left(Q_{k}\left(z^{b}\right) T_{i}(z) \tilde{Q}_{l}\left(z^{b}\right) \tilde{T}_{i}(z)\right) \downarrow_{b . M} \\
& \quad=\delta(k-l), \quad k, l=0, \cdots, M-1
\end{aligned}
$$

i.e.,

$$
\left(Q_{k}(z) \tilde{Q}_{l}(z)\left(T_{i}(z) \tilde{T}_{i}(z)\right) \downarrow_{b}\right) \downarrow_{M}=\delta(k-l) .
$$

But by Lemma 7 ,

$$
\left(T_{i}(z) \tilde{T}_{i}(z)\right) \downarrow_{b}=B(z) \tilde{B}(z)
$$

for some FIR $B(z)$. Substituting in (6.25) we have

$$
\left(Q_{k}(z) \tilde{Q}_{l}(z)(B(z) \tilde{B}(z))\right) \downarrow_{M}=\delta(k-l) .
$$

Define a new set of transfer functions

$$
Q_{i}^{\prime}(z)=Q_{i}(z) B(z) \quad i=0, \cdots, M-1 .
$$

Hence,

$$
\left(Q_{k}^{\prime}(z) \tilde{Q}_{l}^{\prime}(z) \downarrow_{M}=\delta(k-l) \quad k, l=0, \cdots, M-1 .\right.
$$

This means that the functions $Q_{k}^{\prime}(z) k=0, \cdots, M-1$ form a PU-set. By Lemma 4, they cannot have a common factor, except a constant. Equation (6.25) now becomes

$$
\left(Q_{k}^{\prime}(z) \tilde{Q}_{l}^{\prime}(z)\left(T_{i}^{\prime}(z) \tilde{T}_{i}^{\prime}(z)\right) \downarrow_{b}\right) \downarrow_{M}=\delta(k-l)
$$

with

$$
\left(T_{i}^{\prime}(z) \tilde{T}_{i}^{\prime}(z)\right) \downarrow_{b}=1 \text {. }
$$

Note that now

$$
F_{k}(z)=Q_{k}^{\prime}\left(z^{b}\right) T_{i}^{\prime}(z), \quad k=0, \cdots, M-1 .
$$

This proves that the filters on an input level can be made to form a PU-set. In other words, Fig. 14(a) can be redrawn as Fig. 14(c) where the $Q_{k}^{\prime}(z), k=0, \cdots, M-$ 1 form a PU-set.

Lemma 9: Consider Fig. 14(c), drawn alternatively as in Fig. 14(b). Let $F_{m}(z)$ be a set of orthonormal FIR functions satisfying (5.4). Remove an input level of the tree on which the filters formed a PU-set, i.e., remove the filters $Q_{k}^{\prime}(z), k=0, \cdots, M-1$ and the interpolators $(\downarrow M)$ in Fig. 14(c). Then the remaining part of the tree also gives an orthonormal basis.

Proof: We showed in the previous Lemma that the filters $Q_{i}^{\prime}(z) i=0, \cdots, M-1$ constitute a PU-set on one input level of the tree. Removing these filters gives rise to a modified filter bank. We have to prove that the filters in this modified bank give an orthonormal basis, i.e., we have to show that

$$
\left(T_{i}^{\prime}(z) \tilde{T}_{i}^{\prime}(z)\right) \downarrow_{b}=1
$$

and that

$$
\left(T_{i}^{\prime}(z) \tilde{F}_{p}(z)\right) \downarrow_{g 8}=0, \quad g_{8}=\operatorname{gcd}\left(b, I_{p}\right) .
$$

We have proved (6.33) while proving Lemma 8 . Hence we only need to prove $(6.34)$. From the orthonormality of the original basis (Fig. 14(b)), we have

$$
\left(F_{k}(z) \tilde{F}_{p}(z)\right) \downarrow_{g 7}=0 \quad k=0, \cdots, M-1
$$

where $g_{7}=\operatorname{gcd}\left(M b, I_{p}\right)$. Using (6.32), this becomes

$$
\left(Q_{k}^{\prime}\left(z^{b}\right) T_{i}^{\prime}(z) \tilde{F}_{p}(z)\right) \downarrow_{g 7}=0 \quad k=0, \cdots, M-1 .
$$


Now, $g_{8}$ is a factor of $g_{7}$; let $g_{7}=c . g_{8}$, where $c$ has to be a factor of $M$. Hence from the above equation we have

$$
\left(Q_{k}^{\prime}\left(z^{b}\right) T_{i}^{\prime}(z) \tilde{F}_{p}(z)\right) \downarrow_{g 8 . \mathrm{c}}=0 \quad k=0, \cdots, M-1 .
$$

By using the noble identity, this becomes

$$
\begin{gathered}
\left(Q_{k}^{\prime}\left(z^{b / g 8}\right)\left(T_{i}^{\prime}(z) \tilde{F}_{p}(z)\right) \downarrow_{g 8}\right) \downarrow_{c}=0 \\
k=0, \cdots, M-1 .
\end{gathered}
$$

It can be verified that $b / g_{8}$ is indeed an integer, enabling us to write (6.38). Let $b / g_{8}=d$. Then it can also be verified that $d$ and $c$ are relatively prime. Hence using Lemma 6, we get

$$
\left(T_{i}^{\prime}(z) \tilde{F}_{p}(z)\right) \downarrow_{g 8}=0
$$

which completes the proof.

Using the above two lemmas, Theorem 4 is easy to prove.

Proof of Theorem 4: Consider the given filter bank which is known to have been generated by using a tree structure. The functions generated by this tree are given to form an orthonormal basis. Every tree has at least one input level. Using Lemma 8 we know that the filters on this input level can be made to form a paraunitary set. Remove these filters. By Lemma 9, the remaining tree also gives an orthonormal basis. Hence we can repeatedly apply Lemma 8 and Lemma 9 to finally reduce the given tree to a one-level tree. But we know from [16], that for a one level tree, if the functions form an orthonormal basis, the filters have a paraunitary polyphase matrix. This proves Theorem 4.

Note: A corollary similar to Corollary 1 can be proved in this case too. Namely, if an orthonormal basis is generated by a generalized tree-structured filter bank, then the polyphase matrices on all levels have to satisfy (4.38). The proof involves mainly bookkeeping of constants while going over Lemmas 8 and 9 , and we do not reproduce it here.

\section{ImPlementation of Paraunitary Filter Banks}

Perfect-reconstruction QMF filter banks have been studied before [11], [13]. The problem of design and implementation of such filter banks has been addressed by Vaidyànathan and Hoang in [18]. In this paper the authors have described a lattice structure for realizing QMF banks. The resulting filters have a paraunitary polyphase matrix. Fig. 15 shows this lattice structure. This lattice is robust in the sense that the paraunitariness of polyphase matrices is preserved in spite of coefficient quantization. Moreover, the lattice has a hierarchical property, i.e., higher order PU-pairs can be obtained from lower order PU-pairs simply by adding more lattice sections. Another important property of the lattice is that by changing the lattice coefficients we can generate all PU-sets. This property makes the lattice particularly important with reference to orthonormal wavelets. We showed in Section IV that all possible orthonormal wavelet bases could be generated
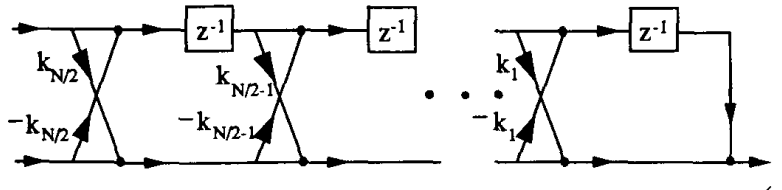

$\mathrm{x}(\mathrm{n})$

Fig. 15. Lattice structure for implementing a two channel synthesis bank with paraunitary polyphase matrix.

using a tree-structural filter bank which had paraunitary matrices on all levels. Thus, if we constructed the treestructure using the above mentioned lattice, we could generate all orthonormal wavelet bases simply by manipulating the lattice coefficients. Moreover, orthonormality would be preserved under coefficient quantization.

Extensions of this structure to $M$-channel filter banks can be found in [11]. Results of Section VI indicate that the $M$-channel lattice could be used to realize the "generalized wavelet bases" mentioned therein.

\section{CONCLUSIONS}

In this paper we have investigated the relationship between orthonormality of wavelet basis and paraunitariness of matrices in a tree-structured filter bank. We started by proving a few interesting results on multirate paraunitary system in Section III. Using these, in Section IV, we showed that a binary tree with paraunitary matrices on all levels (possibly different) generates an orthonormal wavelet basis. More importantly, we proved that all orthonormal bases could be generated by a tree-structured filter bank having paraunitary polyphase matrices on all levels and that the polyphase matrices in fact have to be generalized paraunitary. Knowing the connection between paraunitariness and a special lattice structure, we conclude that all orthonormal wavelet bases could be generated by manipulating the coefficients of the lattice. Hence paraunitariness of polyphase matrices is a necessary and sufficient condition for wavelet orthonormality.

In Section V, we have developed the equations governing orthonormality for general discrete time bases. The relation derived in this section showed that the gcd of the two decimation factors plays a role in the orthonormality equation for two functions.

Using these relations, in section VII, we studied the concept of orthonormality with respect to arbitrary tree structured filter banks. We showed that a tree with paraunitary polyphase matrices gives an orthonormal basis; conversely, a set of orthonormal functions which can be generated using a tree can be generated specifically by a paraunitary tree. This proves the equivalence of paraunitariness and orthonormality in the context of arbitrary tree structures. The generalized tree structure would be convenient in the analysis of waveforms in which the frequency characteristics are not monotonic, as Fig. 11(c) suggests.

\section{APPENDIX}

Consider the orthonormality relation for generalized wavelets (5.2) reproduced below for the sake of conven- 
ience:

$$
\begin{aligned}
& \sum_{n=-\infty}^{\infty} f_{k}\left(n-I_{k} m\right) f_{i}^{*}\left(n-I_{l} i\right) \\
& \quad=\delta(k-l) \delta(m-i) \quad \text { for all integers } m, i .
\end{aligned}
$$

Put $\left(n-I_{k} m\right)=p$. Hence $n=I_{k} m+p$. Therefore, the above equation becomes

$$
\sum_{p=-\infty}^{\infty} f_{k}(p) f_{i}^{*}\left(p-\left(I_{l} i-I_{k} m\right)\right)=\delta(k-l) \delta(m-i) .
$$

Let $g=\operatorname{gcd}\left(I_{k}, I_{l}\right)$. Hence there exists a $j$ such that $\left(I_{l} i\right.$ $\left.-I_{k} m\right)=g j$ for all $m, i$. Also, by Euclid's identity, there exist integers $\hat{I}_{l}$ and $\hat{I}_{k}$ such that $\left(I_{l} \hat{I}_{l}-I_{k} \hat{I}_{k}\right)=g$. Using these two facts, it can be shown that the condition (A.1) is identical to the condition

$$
\sum_{p=-\infty}^{\infty} f_{k}(p) f_{i}^{*}(p-g i)=\delta(k-l) \delta(i) .
$$

where $g$ is the gcd of $\left(I_{k}, I_{l}\right)$. A change of dummy variables results in (5.3).

\section{ACKNOWLEDGMENT}

The authors wish to thank $T$. Chen, graduate student at the California Institute of Technology, for several useful comments.

\section{REFERENCES}

[1] R. Coifman, Y. Meyer, S. Quake, and V. Wickerhauser, "Signal processing with wavelet packets," Numerical Algorithms Research Group, Yale University, 1990.

[2] S. Mallat and W. L. Hwang, "Singularity detection and processing with wavelets," preprint.

[3] R. Kronland-Martinet, "The wavelet transform for analysis, synthesis, and processing of speech and music sounds, " Comput. Music $J$. vol. 12 , no. 4 , pp. $11-20,1988$.

[4] S. Mallat, "A theory for multiresolution signal decomposition: The wavelet representation," IEEE Trans. Patt. Anal. Machine Intell., vol. 11, pp. 674-693, July 1989.

[5] S. Mallat, "Multifrequency, channel decomposition of images and wavelet models," IEEE Trans. Acoust., Speech, Signal Processing, vol. 37, pp. 2091-2110, 1989

[6] F. Tuteur, "Wavelet transformations in signal detection," in Proc. Int. Conf. ASSP, New York, Apr. 1988, pp. 1435-1438.

[7] M. Antonin, M. Barlaud, P. Mathieu, and I. Daubechies, "Image coding using vector quantization in wavelet transform domain." in Proc. Int. Conf. ASSP. Albuquerque, NM, 1990, pp. 2297-2300.

[8] A. Grossman and J. Morlet, "Decomposition of Hardy functions into square integrable wavelets of constant shape," SIAM J. Math. Anal., pp. 723-736, 1984

[9] 1. Daubechies, A. Grossman, and Y. Meyer, "Painless nonorthogonal expansions," J. Math. Phys., pp. 1271-1283, 1986.

[10] I. Daubechies, "Orthonormal bases of compactly supported wavelets," Commun. Pure Appl. Math., vol. 4, pp. 909-996, Nov. 1988.
[11] P. P. Vaidyanathan, "Multirate digital filters, filter banks, polyphase networks, and applications: A tutorial," Proc. IEEE, vol. 78, no. 1, pp. 56-93, Jan. 1990.

[12] O. Rioul and M. Vetterli, "Wavelets and signal processing," IEEE Signal Processing Mag., Oct. 1991.

113] M. J. T. Smith and T. P. Barnwell, III, "Exact reconstruction techniques for tree-structured subband coders," IEEE Trans. Acoust. Speech, Signal Processing, vol. 34, pp. 434-441, June 1986.

[14] P. P. Vaidyanathan, "On power-complementary FIR filters," IEEE Trans. Circuits Syst., vol. 32, pp. 1308-1310, Dec. 1985.

II5] M. Vetterli and C. Herley, "Wavelets and filter banks: Theory and design." Internal Rep., Columbia University, 1990.

[16] P. P. Vaidyanathan, "Lossless systems in wavelet transforms," in Proc. IEEE Int. Symp. Circuits Syst., Singapore, 1991.

[17] H. Zou and A. H. Tewfik, "Design and parametrization of compactly supported wavelets and wavelet packets," Internal Rep., Univ. Minnesota, May 1990.

[18] P. P. Vaidyanathan and P. Q. Hoang, "Lattice structures for optimal design and robust implementation of two-channel perfect reconstruction QMF banks," IEEE Trans. Acoust., Speech, Signal Processing, vol. 36, pp. 81-94, Jan. 1988.

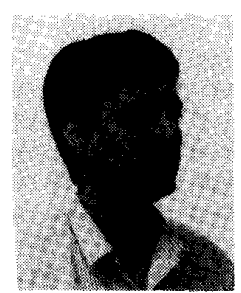

Anand K. Soman (S'91) was born on April 20, 1968 in Bombay. India. He received the B.Tech. degree in electrical engineering from $\mathbf{I}$. I. T. Bombay in 1989 . He is currently pursuing the doctoral degree at the California Institute of Technology, Pasadena, CA. His research interests include multirate signal processing, adaptive filtering, and digital filter design.

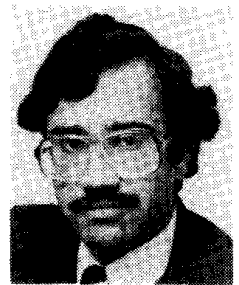

P. P. Vaidyanathan (S'80-M'83-SM'88-F'91) was born in Calcutta, India, on October 16,1954 He received the B.Sc. (Hons.) degree in physics and the B.Tech. and $M$.Tech. degrees in radiophysics and electronics, all from the University of Calcutta, India, in 1974, 1977, and 1979, respectively, and the Ph.D. degree in electrical and computer engineering from the University of California, Santa Barbara, in 1982.

He was a postdoctoral fellow at the University of California, Santa Barbara, from September 1982 to March 1983. In March 1983 he joined the Electrical Engineering Department of the California Institute of Technology as an Assistant Professor, and since 1988 has been an Associate Professor of Electrical Engineering there. His main research interests are in digital signal processing, multirate systems, wavelet transforms, and adaptive filtering.

Dr. Vaidyanathan served as Vice-Chairman of the Technical Program Committee for the 1983 IEEE International Symposium on Circuits and Systems, and as an Associate Editor for the IEEE Transactions ON CIR. CUITS AND SYSTEMS for the period of 1985-1987. He also served as the Technical Program Chairman for the 1992 IEEE International Symposium on Circuits and Systems. He was a recipient of the Award for Excellence in Teaching at the California Institute of Technology for the year 19831984. He also received the NSF's Presidential Young Investigator Award in 1986. In 1989 he received the IEEE ASSP Senior Award for his paper on multirate perfect-reconstruction filter banks. In 1990 he received the S. K. Mitra Memorial Award from the Institute of Electronics and Telecommunications Engineers, India, for his joint paper in the IETE journal. He was elected Fellow of the IEEE in 1991. 